\title{
IDENTIFICACIÓN DE DIFERENTES ZUMOS MEDIANTE NARIZ ELECTRÓNICA PORTÁTIL
}

\author{
Sara González ${ }^{1}$, David Palomeque-Mangut ${ }^{1}$, Juan Álvaro Fernández ${ }^{1}$, José Pedro Santos ${ }^{2}$, Jesús Lozano ${ }^{1}$ \\ ${ }^{1}$ Escuela de Ingenierías industriales. Universidad de Extremadura, Av. Elvas s/n, 06006 Badajoz, España. \\ ${ }^{2}$ Instituto de Tecnologías Físicas y de la Información. Consejo Superior de Investigaciones Científicas, \\ C/ Serrano 144, 28006 Madrid.
}

\begin{abstract}
Resumen
Se describe en esta comunicación una nariz electrónica portátil con conexión wifi para la identificación de aromas. La nariz electrónica es fácil de utilizar y es autónoma gracias a sus baterías. Consta de un sistema de una bomba para la extracción de los aromas, microsensores resistivos y un microcontrolador para el control de todo el sistema. Además tiene conexión a internet y una pantalla táctil a color. Por último, mediante un sistema de reconocimiento de patrones se procesan los datos obtenidos de los sensores para conseguir una clasificación de las muestras en categorías previamente aprendidas. Los resultados muestran que el sistema permita la clasificación de cuatro sabores de zumos diferentes.
\end{abstract}

Palabras Clave: Nariz electrónica, sensores de gases, zumos, reconocimiento de patrones

\section{INTRODUCCIÓN}

Los investigadores comenzaron a explorar el proceso de discriminación de la calidad de los alimentos debido a las enfermedades que se transmitían a través de ellos. La nariz humana está limitada a la hora de la evaluación del olor, por ello surgió la necesidad de crear un instrumento capaz de imitar el sentido humano del olfato.

En 1980, la nariz electrónica fue aceptada como un instrumento que comprende un conjunto de sensores electroquímicos de gas heterogéneos y un sistema de reconocimiento de patrones [1]. Por lo tanto, una vez que se recogen los datos de los sensores, el sistema de reconocimiento de patrones realiza un procesado de los datos para analizarlos y clasificarlos.

Uno de los puntos clave de la fabricación del zumo es detectar la posible contaminación que pueda existir en el zumo en la fase temprana, ya que esta contaminación provoca una pérdida grave en la economía al desperdiciar los productos. La nariz electrónica, que se muestra en la figura 1, puede ser útil para analizar rápidamente el olor en los zumos y para detener lo más temprano posible la contaminación existente [2].

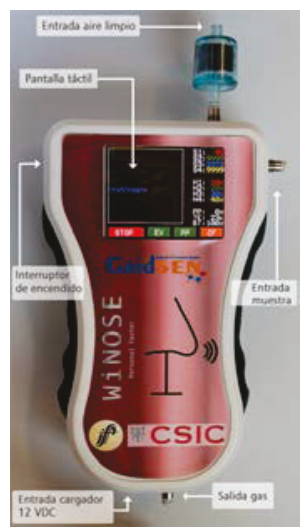

Figura 1: Nariz electrónica

La nariz electrónica al ser fácil de usar, de bajo coste y capaz de proporcionar un procedimiento de análisis rápido, podría ser utilizada como un sistema de evaluación sensorial en la industria de la fruta como alternativa a los métodos tradicionales, tales como la evaluación sensorial por el humano [3]. El método tradicional consume mucho tiempo al personal y puede llegar a ser muy costoso en comparación con el sistema propuesto.

Un estudio con un zumo de manzana [4] resultó que esté podría deteriorarse y causar un defecto sensorial, lo que provocaría quejas de los consumidores. Por ello, mediante una nariz electrónica se propuso la detección del problema, ya que es una herramienta adecuada para un análisis rápido y fiable del sabor de los zumos.

Se han descrito en la bibliografía diferentes aplicaciones de los sistemas de olfato electrónico o narices electrónicas en sectores variados: sanitario [5-8], medioambiente [9, 10], cosmético (análisis de fragancias en jabones y perfumes [11]) y al sector agroalimentario, producción de alimentos y bebidas (pescado [12], aceite de oliva [13], queso [14], leche [15], fruta [16], cerveza [17], vino [18], vinagre [19], café [20], etc.). 


\section{DESCRIPCIÓN DEL DISPOSITIVO}

En esta sección, se ha descrito el dispositivo desarrollado para la identificación de las diferentes muestras. El sistema se puede dividir en las siguientes partes funcionales: el sistema de extracción de aromas, el sistema de detección o sensores, el sistema de instrumentación y control y el sistema de procesado de datos y señal.

\subsection{SISTEMA DE EXTRACCIÓN DE AROMAS}

Dentro de las distintas técnicas de extracción de olores disponibles para una nariz electrónica [21], se ha utilizado la técnica del espacio de cabeza modificada para la extracción de los olores desde viales de vidrio. La técnica de generación del espacio de cabeza consiste en aislar la muestra de agua en forma líquida en un recipiente herméticamente cerrado, en nuestro caso un vial, de manera que los volátiles que son desprendidos por el agua residual queden conservados en la zona superior del recipiente formando así una muestra gaseosa en equilibrio con el líquido, representativa del olor de la mismas. Seguidamente, a través de unas aberturas para la entrada y salida del gas portador se realiza el transporte de los volátiles desprendidos de la muestra hacia los sensores.

\subsection{SENSORES}

El corazón del instrumento son unos micros sensores de gases resistivos. La nariz admite hasta cuatro microsensores integrados en un solo chip. Se han diseñado para este prototipo varias celdas en aluminio para contener diferentes tipos de arrays de sensores: sensores comerciales fabricados por e2v, Cambridge CMOS y otro no comercial, desarrollado por el GRIDSEN en colaboración con el Centro Nacional de Microelectrónica de Barcelona [22]. Estos arrays consisten en cuatro sensores individuales de película delgada de óxido de estaño suspendidos sobre estructuras micro mecanizadas de silicio que contienen una resistencia calefactora $\mathrm{y}$ contactos para medir la resistencia de la película sensible. Cada sensor puede operar a una temperatura distinta hasta un máximo de $500^{\circ} \mathrm{C}$. En la figura 2 se muestran algunas de las celdas fabricadas con sus respectivos sensores.

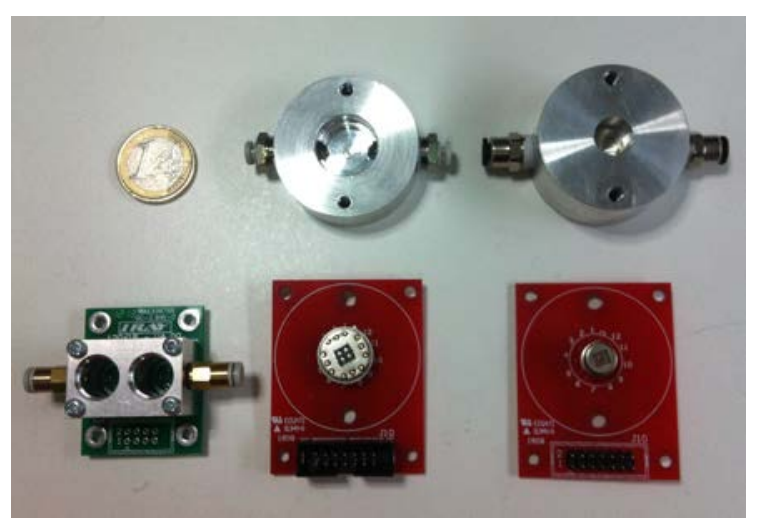

Figura 2: Celdas de los sensores. Izquierda: e2v, centro: sensor CNM, derecha: sensor MICROSENS

La celda de los sensores está conectada a una electroválvula de tres vías (SMC S70_ES) que permite seleccionar entre dos entradas: una para la muestra a medir y otra para un gas de referencia. En esta última se suele poner un filtro de carbón activo. Aguas abajo de la celda se encuentran los sensores de humedad relativa y temperatura (Sensirion SHT15), una microbomba (Rietschle Thomas model 2002) y un caudalímetro (SMC PFMV5). En la tabla 1 se muestran los rangos de medida de los distintos sensores.

Tabla 1: Rango de medida de los sensores.

\begin{tabular}{|l|l|}
\hline Parámetro & Rango \\
\hline Resistencia sensores & $10-5 \times 10^{7}$ ohmios \\
Humedad relativa & $0-100 \%$ \\
Temperatura & $-40-124{ }^{\circ} \mathrm{C}$ \\
Caudal & $0-500 \mathrm{~mL} / \mathrm{min}$ \\
\hline
\end{tabular}

\subsection{SISTEMA DE INSTRUMENTACIÓN Y CONTROL}

En la figura 3 se muestra un esquema de la electrónica del sistema. El sistema es gobernado por un microcontrolador con $16 \mathrm{~Kb}$ de RAM y $128 \mathrm{~Kb}$ de memoria FLASH. Posee varios conversores analógio-digital para la medida de los sensores y varias salidas con modulación de anchura de pulso (PWM) para la calefacción de los sensores. Los principales parámetros de medida así como una gráfica con el histórico de los últimos valores se muestran en una pantalla LCD táctil, lo cual permite también la selección de diferentes parámetros de configuración. Se dispone de un transmisor WIFI para las comunicaciones inalámbricas. La resistencia de los sensores es medida mediante un circuito electrónico basado en un amplificador operacional. Debajo de la placa electrónica de control se ubican las baterías recargables ( 3 x $4500 \mathrm{mAh}$ ) que proporcionan una autonomía de unas ocho horas al sistema. 


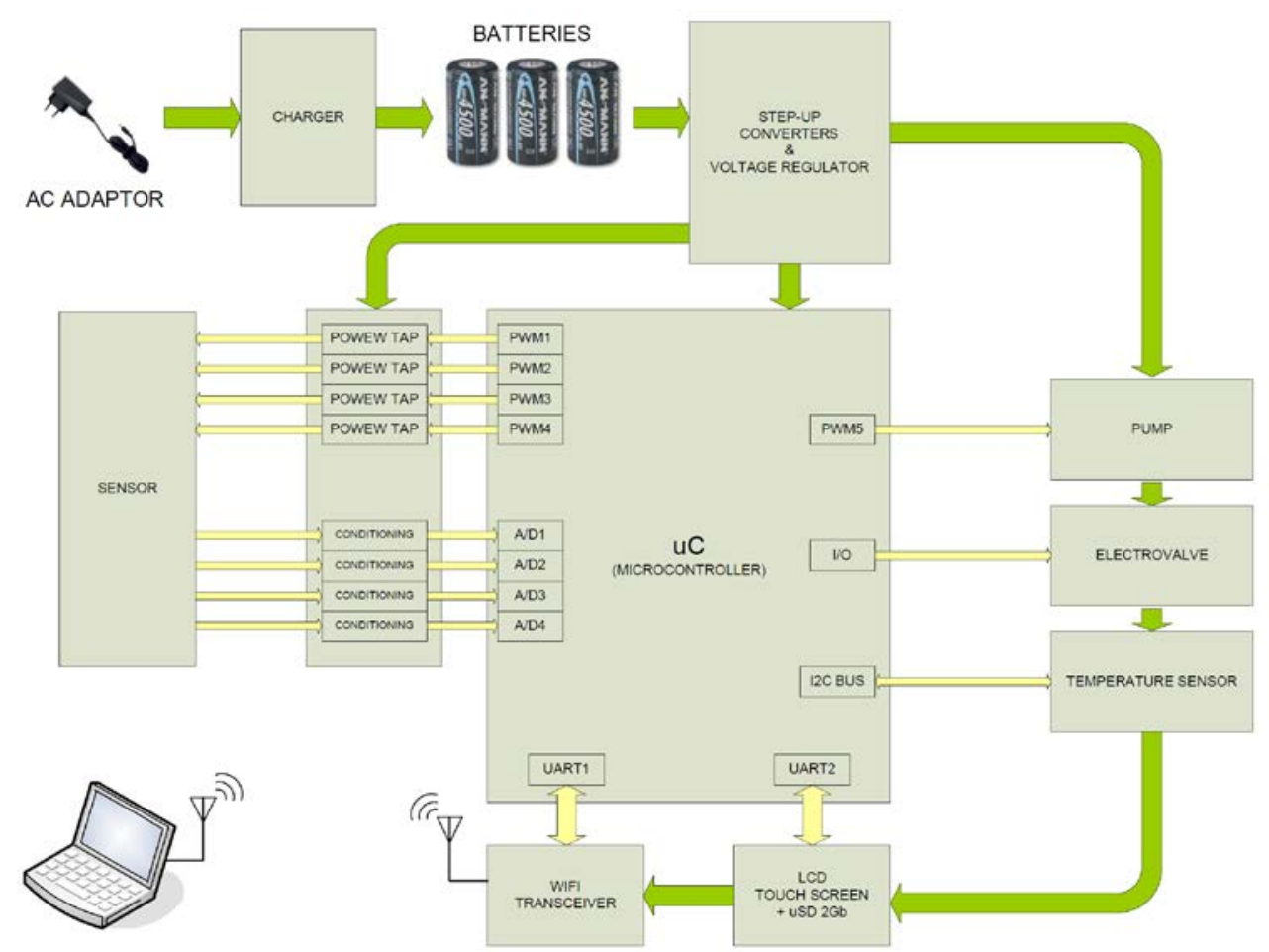

Figura 3: Esquema electrónico del sistema diseñado

La nariz se controla a través de un programa desarrollado en Testpoint, un lenguaje de programación orientado a objeto. Una captura de pantalla de la interfaz de usuario se muestra en la figura 4. En el gráfico inferior se muestran las resistencias de los sensores de gases. En la parte superior se muestran las respuestas. Estas se calculan como la razón de resistencias entre el gas de referencia y la muestra. En esta interfaz simplificada se programan el número de muestras a medir y los tiempos de conmutación de la electroválvula. Existe otra pantalla donde se pueden modificar los valores iniciales de la medida (tiempo entre medidas, calefacción de los sensores y caudal).

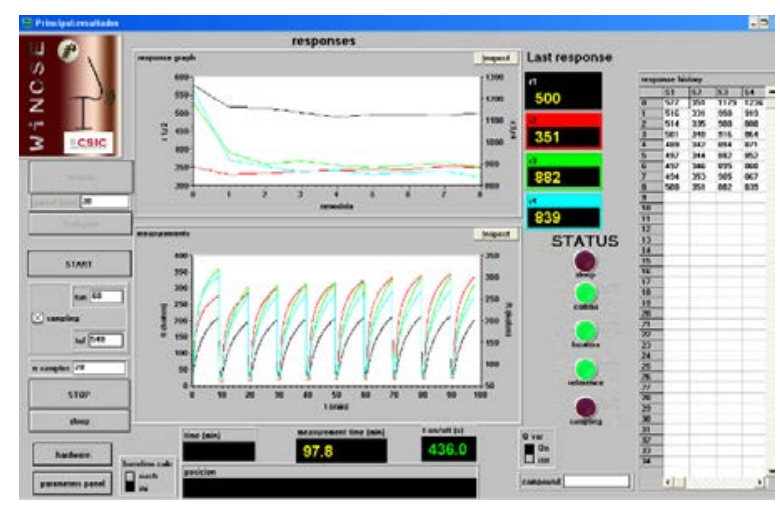

Figura 4: Interfaz de usuario

\subsection{PROCESADO DE LA SEÑAL}

Una de las piezas angulares en un sistema olfativo artificial la constituye el conjunto de técnicas que tratan la información obtenida a través de la matriz de sensores químicos de estado sólido. El procesado de señal multivariable que se integra en las narices electrónicas actuales tiene su origen en la química analítica, también denominada "quimiometría".

El procesado de los datos comienza después de que las señales de los sensores han sido adquiridas y almacenadas en el ordenador. Los datos se guardan en ficheros de texto, con separación de los datos por comas. De esta forma, se almacena el tiempo de la medida, las respuestas de los sensores y otros parámetros como temperatura ambiente, estado de las electroválvulas y caudalímetros, etc. El objetivo principal del procesado de señal es extraer la información relevante para obtener la respuesta deseada, minimizando las interferencias que pueda introducir la matriz de sensores. Las respuestas de los sensores pueden ser utilizados como una "huella electrónica" para caracterizar un amplio rango de olores o compuestos orgánicos volátiles utilizando un sistema de reconocimiento de patrones. Este proceso puede ser dividido en cuatro etapas: extracción de características y pre-procesado de la señal, reducción de la dimensionalidad, clasificación y toma de decisión [23]. Los programas han sido realizados con Matlab y se ha utilizado las toolboxes de Signal Processing y Neural Networks. 
El pre-procesado de los datos medidos de los sensores se ha realizado mediante el algoritmo de resistencia relativa $(\mathrm{RR})$ en el cual la respuesta de cada sensor se calcula dividiendo el valor estacionario de la respuesta de cada sensor frente al valor del gas de referencia. La siguiente etapa tiene como objetivo reducir la dimensionalidad de los datos extraídos. Se utilizará para este fin el análisis de componentes principales (PCA). El PCA es una potente técnica de reconocimiento de patrones, no supervisada y lineal basada en la expansión de Karhunen-Loeve [24]. El método PCA reduce la redundancia o correlación que exista en las respuestas de los sensores. Normalmente la ndimensionalidad del problema se reduce a los dos primeros auto vectores (primeras dos componentes principales) que contienen la máxima información [25-27].

El objetivo final del sistema de Reconocimiento de Patrones diseñado en este trabajo es el etiquetar de forma automática patrones de los cuales desconocemos su clase, es decir: clasificación. Para este tipo de tareas, hace tiempo que las redes neuronales artificiales ocupan un lugar de privilegio. Este tipo de algoritmos, cuya topología está inspirada en las redes neuronales biológicas, ajustan sus modelos de forma experimental, sin necesitar un conocimiento previo de las características de los datos que debe procesar ni de los mecanismos que los producen. Además, su forma matemática de operar no está restringida a relaciones y procesos lineales.

Para la implementación de los algoritmos de las tareas de clasificación se ha utilizado una red feedforward con aprendizaje backpropagation (una red con funciones de base radial) y un clasificador basado en lógica borrosa.

El perceptrón multicapa es una red neuronal artificial (RNA) formada por múltiples capaz, esto le permite resolver problemas que no son linealmente separables. El perceptrón multicapa puede ser totalmente o localmente conectado. En el primer caso cada salida de una neurona de la capa " $i$ " es entrada de todas las neuronas de la capa " $i+1$ ", mientras que en el segundo cada neurona de la capa "i" es entrada de una serie de neuronas (región) de la capa "i+1". Las capas pueden clasificarse en tres tipos: capa de entrada (constituida por aquellas neuronas que introducen los patrones de entrada en la red, en nuestro caso los valores de los sensores), capas oculta (formada por aquellas neuronas cuyas entradas provienen de capas anteriores y cuyas salidas pasan a neuronas de capas posteriores) y capa de salida (neuronas cuyos valores de salida se corresponden con las salidas de toda la red e indican el valor de clasificación de la misma). La propagación hacia atrás del error (también conocido como regla delta generalizada), es el algoritmo generalmente utilizado en el entrenamiento de estas redes.

Las redes de base radial son un tipo de redes de neuronas artificiales que calculan la salida de la función en función de la distancia a un punto denominado centro. Al igual que con los perceptrones multicapa, sirven como aproximadores universales. La función de base radial es una función que calcula la distancia euclidea de un vector de entrada $\mathrm{x}$ respecto de un centro $\mathrm{c}$, de tal manera que resulta la siguiente función:

$$
f(\mathbf{x})=\left(\left\|\mathbf{x}-\mathbf{c}_{i}\right\|\right)
$$

A cada neurona de la capa de entrada le corresponde una función de base radial $\Phi(\mathbf{x})$ y un peso de salida wi. El patrón de salida ingresa a una neurona de salida que suma las entradas y da como resultado una salida. La función de una red RBF final resulta:

$$
F(\mathbf{x})=\sum_{i=1}^{N} w_{i} \Phi\left(\left\|\mathbf{x}-\mathbf{c}_{i}\right\|\right)
$$

Las redes RBF tienen una construcción rígida de tres capas: Capa de entrada, capa oculta y capa de salida.

Por otra parte, esta clasificación de muestras también puede llevarse a cabo mediante Lógica Borrosa (LB) o Fuzzy. La LB es una extensión matemática de la Lógica Clásica, más restrictiva. En Lógica Clásica, todo conjunto puede definirse como una lista (finita o infinita) no ordenada de elementos, $\mathrm{A}=\{\mathrm{aj}\}$, donde cada uno de sus elementos aj pertenecen al conjunto $\mathrm{A}$, y por tanto no pertenecen a cualquier posible subconjunto fuera de A (conjunto complementario de A). Es decir, un elemento no puede pertenecer y no pertenecer al mismo tiempo a un conjunto cualquiera. Sin embargo, en LB se permite que un conjunto borroso pueda asignar un grado de pertenencia a sus elementos miembros, es decir, un elemento puede pertenecer parcialmente a un conjunto $\mathrm{A}$, y por tanto también parcialmente a su complementario. Normalmente, se asigna un valor 1 a objetos que pertenecen completamente al conjunto, y un valor 0 a los que están completamente fuera del mismo. El número real $\mu$ asignado al objeto se identifica como su grado de pertenencia al conjunto. En la presente aproximación de reconocimiento de patrones, se utilizan conceptos de LB para construir un clasificador borroso. Este módulo se diseña de forma que cada clase sea clasificada como una tupla $\langle\mu$, L $>$, donde $\mathrm{L}$ es una etiqueta lingüística que identifica la clase (en este caso el tipo de zumo), y $\mu$ es el grado numérico (no lingüístico) de pertenencia a L. En general, $\mu$ se implementa para un sensor concreto como una función sobre el rango de posibles valores detectados por el mismo, $\mathrm{x}$, de forma que pueda 
modelarse una función conocida como función de pertenencia, $\mu(\mathrm{x})$, de acuerdo a los valores reales proporcionados por cada uno de los sensores de la nariz electrónica ante las distintas muestras a clasificar [28].

Las reglas $\mathrm{LB}$ se forman como combinación de funciones de pertenencia sobre distintas variables, como resultado de una operación lógica entre conjuntos asociados a sensores individuales. Desde el punto de vista del clasificador borroso, cada regla de clasificación suele ser el resultado de una operación borrosa AND sobre los valores proporcionados por un número, en principio variable, de sensores (al menos uno). Dado que cada regla intenta seleccionar una respuesta característica de los sensores de la nariz electrónica a una sustancia particular, se puede construir una regla borrosa para cada conjunto de muestras a clasificar [29].

Para determinar la validez de los modelos de clasificación con redes neuronales y lógica difusa es necesario realizar predicciones de medidas de las que se sabe el valor correcto de salida para poder comparar la estimación realizada con el valor real. Este paso final en el reconocimiento de patrones permite la estimación de errores o rendimientos del modelo entrenado mediante técnicas de validación. En este trabajo, debido a la dificultad de obtener una gran cantidad de medidas en algunos de los experimentos y para sacar más rendimiento a las medidas obtenidas, se han utilizado validaciones cruzadas de orden 1 (también denominadas leaveone-out), lo que supone haber generado tantas redes como medidas se disponen. Esta filosofía no está pensada para proponer un modelo concreto para la clasificación de muestras o para la predicción de cada parámetro, sino para probar la validez de la aproximación.

\section{RESULTADOS}

Las muestras que se han utilizado para el proceso de medición han sido zumos de cuatro sabores diferentes. Estos sabores son: melocotón, piña, naranja y tomate. Antes de comenzar el proceso, el baño térmico, utilizado para mantener a una temperatura constante las muestras, ha sido llenado con agua y programado para una temperatura de $16^{\circ}$. Una vez que el baño térmico ha alcanzado la temperatura deseada, se ha procedido a la medición de las muestras. En total, se han obtenido 60 mediciones, es decir, 15 medidas por cada sabor de zumo. Cada muestra era 10 mililitro del zumo en cuestión. El tiempo completo del ciclo de medida ha sido 10 minutos: en primer lugar, el sensor está durante 1 minuto midiendo la muestra $y$ seguidamente, el sensor tiene un tiempo de recuperación de 9 minutos. Este proceso se repite para un total de las 60 muestras. La medición de las muestras se realizaron con un caudal de $150(\mathrm{~L} / \mathrm{min})$ y las temperaturas de operación de los sensores han estado entre 300 y $400^{\circ} \mathrm{C}$.

Terminada la obtención de todas las mediciones de las muestras, estás mismas son almacenadas en disco para proceder posteriormente a su análisis. El objetivo final del proceso es determinar si el sistema es capaz de identificar los diferentes zumos. Para ello, en primer lugar se va a realizar el Análisis de Componentes Principales y así mostrar la distribución de las medidas en un gráfico. En la figura 5, se representa el PCA de las mediciones de las cuatro muestras de zumos y se puede observar que las zonas correspondientes a las medidas de los diferentes zumos están separadas entre sí, con lo que se podría asegurar que el sistema ofrece diferente respuesta a las diferentes muestras.

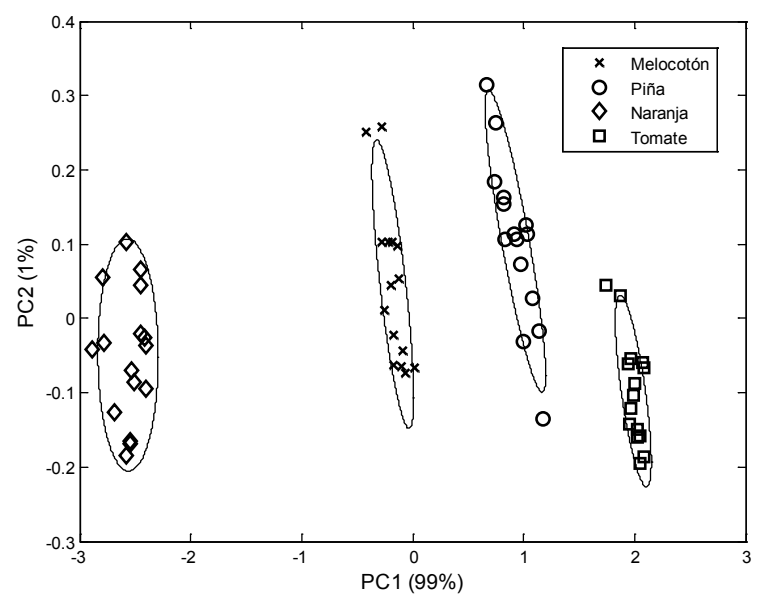

Figura 5: Análisis de Componentes Principales de las medidas de las muestras analizadas

A partir de la observación del PCA, se observa una alineación de los puntos de cada clase, es decir una variación temporal de los datos obtenidos, conocido como deriva en los sensores, que va aumentando a medida que se suceden las medidas. Para corregir esta deriva, se modela el efecto del tiempo sobre la medida arrojada por el sensor a través de la Ecuación (3).

$\mathrm{S}_{\mathrm{X}}=\alpha_{1} \mathrm{t}+\alpha_{0}+\varepsilon$

$S_{X}$ es la respuesta del sensor $\mathrm{X}, t$ es el tiempo que transcurre desde la primera medida, $\alpha_{0}$ es el valor de la primera medida, $\alpha_{1}$ es la variación de la respuesta del sensor por unidad de tiempo y $\varepsilon$ es la variabilidad entre muestras con causas no asignables, que se supone que sigue una distribución normal de media 0 y varianza $\sigma(\varepsilon \sim \mathrm{N}(0, \sigma))$.

Mediante una regresión lineal de primer orden, se pueden a ajustar los resultados obtenidos a una recta 
cuya expresión matemática se presenta en la Ecuación 4.

$S^{\prime}{ }_{X}=\alpha_{1} t+\alpha_{0}$

Una vez obtenidos los coeficientes de la regresión lineal simple, se podrá corregir la deriva de los sensores realizando la operación mostrada en la Ecuación 5.

$\mathrm{S}_{\mathrm{X}, \mathrm{corr}}=\left(\mathrm{S}_{\mathrm{X}}-\left(\mathrm{S}^{\prime}{ }_{\mathrm{X}}-\alpha_{0}\right)=+\alpha_{0}+\varepsilon\right.$

$S_{X, \text { corr }}$ corresponde a la respuesta del sensor X corregida mediante una regresión lineal simple, puesto que su expresión se reduce a la salida del sensor en la primera medida más la variabilidad no asignable.

En la figura 6, se representan las mediciones de las cuatro muestras de zumos con la corrección de deriva efectuada, se puede observar como la agrupación de las diferentes medidas es mayor en cada una de las clases.

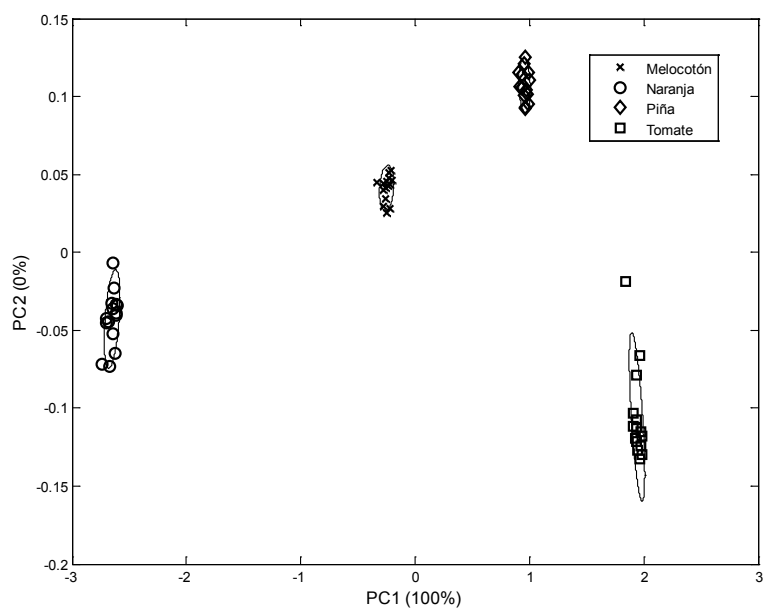

Figura 6: Análisis de Componentes Principales de las medidas corregidas de las muestras analizadas

Los resultados obtenidos en el PCA son confirmados con la clasificación mediante Redes Neuronales y Lógica Difusa, en el que se ha obtenido un 100\% de éxito en la clasificación, es decir todas las muestras han sido correctamente clasificadas. En la tabla 2 se muestra la matriz de confusión obtenida en la validación utilizando el método Leave One Out.

Tabla 2: Matriz de confusión.

\begin{tabular}{|l|c|c|c|c|}
\hline & Melocotón & Piña & Naranja & Tomate \\
\hline Melocotón & 15 & 0 & 0 & 0 \\
\hline Piña & 0 & 15 & 0 & 0 \\
\hline Naranja & 0 & 0 & 15 & 0 \\
\hline Tomate & 0 & 0 & 0 & 15 \\
\hline
\end{tabular}

Las filas representan las muestras reales, mientras que las columnas indican las muestras del sistema y podemos observar en la tabla 2 que el sistema clasifica perfectamente las 15 muestras reales.

\section{CONCLUSIÓN}

Como conclusión obtenemos que el sistema de la nariz electrónica es capaz de identificar los cuatro aromas de zumo con las técnicas empleadas, además esta técnica podría ser utilizada también con otras bebidas. Las ventajas que aporta también este sistema son que es de bajo coste, que al ser de pequeño tamaño es posible transportarla a cualquier lugar y su gran autonomía.

\section{Agradecimientos}

Este trabajo ha sido realizado gracias al apoyo del Ministerio de Economía y Competitividad a través del proyecto TEMINAIR (TEC2013-48147-C6).

\section{Referencias}

[1] J.W. Gardner, P.N. Bartlett, "A brief history of electronic noses". Sensors and Actuators B, vol. 18-19 (1994), p. 211-220

[2] Wang, H. X., Hu, Z. Q., Long, F. Y., Guo, C. F., Yuan, Y. H., (2016) "Early detection of Zygosaccharomyces rouxii-spawned in apple juice by electronic nose combined with chemometrics", International Journal of Food Microbiology, 217, pp. 68-78.

[3] Qiu, S. S., Wang, J., (2015) “Application of Sensory Evaluation, HS-SPME GC-MS, ENose, and E-Tongue for Quality Detection in Citrus Fruits", Journal of Food Science, 80(10), pp. S2296-S2304.

[4] Huang, X. C., Yuan, Y. H., Wang, X. Y., Jiang, F. H., Yue, T. L., (2015) "Application of Electronic Nose in Tandem with Chemometric Analysis for Detection of Alicyclobacillus acidoterrestris-Spawned Spoilage in Apple Juice Beverage", Food and Bioprocess Technology, 8(6), pp. 1295-1304.

[5] M. Falasconi, E. Gobbi, M. Pardo, M. Torre, A. Bresciani and G. Sberveglieri, "Detection of toxigenic strains of Fusarium verticillioides in corn by electronic olfactory system". Sensors and Actuators B: Chemical, vol. 108, Issues 12, 22 July 2005, pp. 250-257.

[6] C. Di Natale, A. Macagnano, E. Martinelli, R. Paolesse, G. D'Arcangelo, C. Roscioni, A.Finazzi-Agrò and A. D'Amico, "Lung cancer identification by the analysis of breath by means of an array of non-selective gas sensors". Biosensors and Bioelectronics, vol. 18 (2003), Issue 10, pp. 1209-1218 
[7] Y. Lin, H. Guo, Y. Chang, M. Kao, H. Wang and R. Hong, "Application of the electronic nose for uremia diagnosis", Sensors and Actuators B: Chemical, vol. 76 (2001), Issues 13, pp. 177-180

[8] J.W. Gardner, H.W. Shin and E.L. Hines, "An electronic nose system to diagnose illness", Sensors and Actuators B: Chemical, vol. 70 (2000), Issues 1-3, pp. 19-24

[9] J.P. Viricelle, C. Pijolat, R. Lalauze,"Application of chemical sensors to atmosferic pollution". Proceedings of the 14th European Conference on Solid-State Transducers (EUROSENSORS XIV). Copenhagen (Denmark), p. 87-90. (2000).

[10] S. Zampolli, I. Elmi, F. Ahmed, M. Passini, G.C. Cardinali, S. Nicoletti, L. Dori, "An electronic nose based on solid state sensor arrays for low-cost indoor air quality monitoring applications". Sensors \& Actuators B, Vol. 101 (2004), pp. 39-46.

[11] A. Branca, P. Simonian, M. Ferrante, E. Novas, R. Martín, "Electronic nose based discrimination of a perfumery compound in a fragrance", Sensors and actuators B, vol. 92 (2003), pp. 222-227.

[12] G. Olafsdottir et al. "Multisensor for fish quality determination" Trends in Food Science \& Technology, Vol. 15 (2004), pp. 86-93.

[13] A. Taurino , S. Capone , C. Distante , M. Epifani, R. Rella, P. Siciliano, "Recognition of olive oils by means of an integrated sol-gel $\mathrm{SnO} 2$ Electronic Nose" Thin Solid Films 418 (2002), pp. 59-65.

[14] J. Bargon et al. "Determination of the ripening state of Emmental cheese via quartz microbalances" Sensors and Actuators B, Vol. 95 (2003), pp. 6-19

[15] K. Brudzewski, S. Osowski, T. Markiewitcz, "Classification of milk by means of an electronic nose and SVM neural network" Sensors \& Actuators B, Vol. 98 (2004), pp. 291-298.

[16] S. Saevels, J. Lammertyn, A. Berna, E. Veraverbeke, C. Di Natale and B. M. Nicolaï, "An electronic nose and a mass spectrometrybased electronic nose for assessing apple quality during shelf life" Postharvest Biology and Technology, Vol. 31 (2004), pp. 9-19.

[17] J. Lozano, J. Pando, J.P. Santos, M.D. Moreno, J.A. Fernández, "Discriminación de cervezas artesanales extremeñas mediante nariz electronica portátil" Actas del XI Simposio CEA de Control Inteligente (2015), pp. 69-74.

[18] J. Lozano, J.P. Santos, M.C. Horrillo, (2016) "Wine Applications With Electronic noses". En "Electronic noses and Tongues in Food Science" Academic Press, pp. 137-148.
[19] E. Anklam, M. Lipp, B. Radovic, E. Chiavaro and G. Palla, "Characterisation of Italian vinegar by pyrolysis-mass spectrometry and a sensor device ('electronic nose')" Food Chemistry, Vol. 61 (1998), pp. 243-248.

[20] M. Pardo, G. Niederjaufner, G. Benussi, E. Comini, G. Faglia, G. Sberveglieri, M. Holmberg and I. Lundstrom, "Data preprocessing enhances the classification of different brands of Espresso coffee with an electronic nose" Sensors and Actuators B, Vol. 69 (2000), pp. 397-403.

[21] Lozano, J., Santos, J. P., Gutiérrez, J., Horrillo, M. C., (2007) "Comparative study of sampling systems combined with gas sensors for wine discrimination", Sensors and Actuators B: Chemical, 126(2), pp. 616-623.

[22] Gracia, L., Santander, J., Cané, C., Horrillo, M. C., Sayago, I., Gutierrez, J., (2001) "Results on the reliability of silicon micromachined structures for semiconductor gas sensors", Sensors and Actuators B: Chemical, 77(1), pp. 409-145.

[23] Gutierrez-Osuna, R., (2002) "Pattern analysis for machine olfaction: a review", Sensors Journal, IEEE, 2(3), pp. 189-202.

[24] Kittler, J., Young, P. C., (1973) “A new approach to feature selection based on the Karhunen-Loeve expansion”, Pattern recognition, 5(4), pp. 335-352.

[25] McAlernon, P., Slater, J. M., Lowthian, P., Appleton, M., (1996) "Interpreting signals from an array of non-specific piezoelectric chemical sensors", Analyst, 121(6), pp. 743-748.

[26] Nanto, H., Tsubakino, S., Ikeda, M., Endo, F., (1995) "Identification of aromas from wine using quartz-resonator gas sensors in conjuction with neural-network analysis", Sensors and Actuators B: Chemical, 25(1), pp. 794-796.

[27] Rose-Pehrsson, S. L., Grate, J. W., Ballantine Jr, D. S., Jurs, P. C., (1988) "Detection of hazardous vapors including mixtures using pattern recognition analysis of responses from surface acoustic wave devices", Analytical chemistry, 60(24), pp. 2801-2811.

[28] Loutfi, A., Coradeschi, S., (2008) "Odor recognition for intelligent systems", IEEE Trans. Intelligent Systems, 23(1), pp. 41-48.

[29] Kosko, B., (1997) "Fuzzy Engineering, Prentice Hall, Upper-Saddle River”, NJ, USA. 